\title{
Belly Dancer Dyskinesia During Pregnancy: Case Report from Harar, Eastern Ethiopia
}

\section{Tadesse Gure (ID}

Department of Gynecology and Obstetrics, Haramaya University, Harar, Ethiopia
Correspondence: Tadesse Gure

Tel +2519138687/4

Email tadebuna@yahoo.com
Introduction: Belly dancer dyskinesia (BDD) is an involuntary waving and writhing type of abdominal wall movement, which resemble those of a belly dancer. Worldwide only four cases of belly dancer dyskinesia during pregnancy were reported. In two of the reported cases, it has recurred in the succeeding pregnancy while the remaining one did not mention about the recurrence. I am reporting a 25 year old primigravida woman with gestational age of 25 weeks plus 6 days presented with involuntary, rhythmic and painful abdominal movement of three weeks duration and diagnosed as belly dancer dyskinesia after investigations. The abnormal body movement disappeared after delivery and did not recur in the second pregnancy.

Conclusion: "Belly Dancer Dyskinesia" during pregnancy is a very rare type of disease with unknown etiology and a little known effective treatment against it.

Keywords: belly dancer dyskinesia, pregnancy, case report, Ethiopia, Harar

\section{Introduction}

Belly dancer dyskinesia (BDD) in pregnancy is a very rare type of involuntary abdominal movement. Upon reviewing literature, I was able to find only four BDD cases. ${ }^{1-3}$ In two of the literature, they had observed the recurrence of the dyskinesia in the subsequent pregnancy; of which one reported the recurrence in the second month of the following pregnancy. Though the exact causes are unknown, some suggested that the possible etiology of the BDD during pregnancy is that the gravid uterus and hemodynamic (vascular) changes during pregnancy will have a compression effect to the thoracic cord and nerve roots leading to abnormal movement. ${ }^{3}$ Though this suggestion is true for pregnant mothers of second and third trimesters it is not true for early trimester pregnancy. For example, a patient with recurring belly dancer dyskinesia at eight weeks of pregnancy is less likely to be caused by uterine pressure effect. ${ }^{2,3}$ For this reason, the exact cause of belly dancer dyskinesia during pregnancy is unknown.

\section{Case Summary}

A 25 year old primigravida woman whose gestational age from LNMP was 25 weeks plus six days presented for the first time with a chief complaint of involuntary abdominal movement of three weeks duration. She stated that three weeks prior to her presentation to the clinic she had experienced involuntary, rhythmic and painful abdominal movement (Supplementary Video 1) (Supplementary Video 2) which started suddenly and stays for 30-120 seconds and happens 3-5 times per day. 
Most of the time, it starts while she was eating food and stops when she was breathing deeply and distracted. Other than these she did not find any predisposing, exacerbating or relieving factors. She presented to the antenatal clinic with these complaints and on arrival the obstetrician had also noticed the rhythmic involuntary abdominal movement as showed in the Supplementary Videos 1 and 2. She denied the use of any medication preceding the involuntary abdominal movement except ferrous sulphate given during ANC visit. She never had any gynecologic and surgical procedure before. Different laboratory and imaging investigations were done for her. These are renal function test, liver function test, thyroid function test, electrolytes, HIV, Venereal Disease for Research Laboratory (VDRL) and CBC and all were within the normal limit. Ultrasound examination revealed 27 weeks singleton intrauterine pregnancy without any abnormal finding. Imaging like MRI, EEG and EMG was not done since it is not available in this locality (Harar) and it was difficult for her to go to the capital city of the country (Addis Ababa) which is $\mathbf{5 8 0}$ kilometers away from Harar town where these imaging services are available.

On physical examination vital signs and all the system examination were unremarkable except the abdominal examination which shows a 26 week sized gravid uterus with positive fetal heartbeat. She was diagnosed with belly dancer dyskinesia and diazepam was prescribed but she refused to take it.

At 38 weeks plus five days she was diagnosed with preeclampsia with severity features after she presented with severe headache of two days duration and her BP was $150 / 100 \mathrm{mmHg}$ for which cervical ripening and induction was started. After 10 hours of total induction time there was no contraction, so she was taken to operation theater and undergone cesarean section for the indication of failed induction to result in delivery of a 4000 $\mathrm{g}$ female live neonate who cried immediately after delivery. She was discharged after 48 hours post-cesarean section hospital stay with good post-partum condition and a healthy neonate.

During the post-partum period, she did not have this abnormal movement and 6 months after her first delivery, she came to the clinic for antenatal follow-up after she had an unplanned but wanted pregnancy. Her second pregnancy antenatal follow-up was uneventful without any abnormal symptom unlike the previous pregnancy. At 39 weeks of pregnancy from her early ultrasound she underwent elective cesarean section for the indication of short inter-pregnancy interval and previous cesarean section.
After two postpartum days of hospital stay, the mother and neonate were discharged healthy.

\section{Discussion}

Belly dancer dyskinesia (BDD) refers to a rare type of abnormal abdominal wall movement, which starts involuntary, visible by the naked eye, slow, semi-continuous, resulting from repetitive, involuntary and often rhythmic contractions of the abdominal wall and/or diaphragm leading to waving and writhing abdominal movements, with slow multidirectional displacement of the umbilicus, resembling those of a belly dancer. ${ }^{4}$ This syndrome is also known as belly dancer syndrome (BDS), diaphragmatic flutter, diaphragmatic myoclonus, abdominal myoclonus and abdominal dystonia. ${ }^{5,6}$ Our patient has similar presentation of the description mentioned above.

The clinical feature of involuntary abdominal wall movement which is rhythmic, repetitive, and dyskinetic was first introduced in 1990 as "belly dancer's dyskinesia" by Iliceto et al. ${ }^{4}$ Though the terminology is repeatedly used since then, the exact prevalence, etiology and treatment of this dyskinesia or syndrome is not well known.

Belly dancer dyskinesia in pregnancy is a very rare type of involuntary abdominal movement, upon reviewing different literature I found only four BDD cases. ${ }^{1-3}$ In two of the literature they have observed the recurrence of the dyskinesia in the subsequent pregnancy and one of them reported at the second month (eight weeks) of the subsequent pregnancy. Though the exact cause is unknown it was suggested that the possible etiology of the BDD during pregnancy is because the gravid uterus and hemodynamic (vascular) changes during pregnancy will have compression effect to the thoracic cord and nerve roots leading to abnormal movement. ${ }^{3}$ Even though this proposed suggestion is true for pregnant mothers at second and third trimesters, it is not true for mothers developing BDD in early trimester. As an example, in one of the patients with recurrent BDD, she experienced this abnormal involuntary abdominal movement at eight weeks of her second pregnancy at which pressure effect of gravid uterus to compress thoracic nerve root is less likely and different imaging of spinal cord during pregnancy and post-partum period also did not reveal any cause., ${ }^{2,3}$ Similar to the previously reported cases, in this patient the exact cause of the BDD is not known. However, unlike the two cases of BDD during pregnancy in this patient it did not recur in the second pregnancy. 
The etiology of this abdominal dyskinesia in the general population is still unclear but some studies suggest that the etiology might be associated with central or peripheral nervous system, some drugs, and electrolyte imbalances because of diarrhea.

One of the differential diagnoses for this abnormal abdominal wall movement is spinal myoclonus (SM). $\mathrm{SM}$ is one of the involuntary types of abdominal movement caused by different disease entities affecting the spinal cord. If thoracic or upper lumbar segment of the spinal cord is involved the feature of the SM mimics that of the belly dancer dyskinesia. This segmental involvement of abdominal contraction is not necessarily originated from spinal segment, it may result from a cerebral problem. ${ }^{4}$

Few studies reported association of BDD with some medications like antipsychotics and dopamine antagonists. Among these drugs causing belly dancer dyskinesia are Quetiapine (typical antipsychotic), Clebopride (dopamine antagonist), etc. ${ }^{7}$

In one case report, they have reported that BDD resulted from central pontine and extra pontine myelinolysis, after rapid correction of hyponatremia. ${ }^{8}$

There is no gold standard investigation modality which can help in the diagnosis of underlying causes for belly dancer dyskinesia. However, different investigation modalities (blood tests like: creatinine, BUN, liver function tests, electrolyte and imaging like: ultrasound, CT-scan (computed tomography) EEG (electroencephalography), MRI, EMG, fluoroscopy and echocardiography) has been done without remarkable finding in different case reports. ${ }^{9}$ In this case also different laboratory investigation was done without any remarkable finding but other imaging was not done since it is not available in this part of the country.

Once diagnosis of BDD is made its treatment is challenging. Though different treatment modalities have been proposed, none of them has been proven to be best effective and safe management modalities for belly dancer dyskinesia. Treatment modalities include both surgical and medical. Among medications used for treatment of BDD are included benzodiazepines, ${ }^{4,9}$ trihexyphenidyl, ${ }^{4}$ valproate, ${ }^{4}$ carbamazepine, ${ }^{10}$ transcutaneous electrical nerve stimulation, ${ }^{11,12}$ and deep brain stimulation. ${ }^{1}$ In one of the literature, BTX injections under ultrasound guidance was given to four patients with $\mathrm{BDD}$ and they found that it is effective in treatment of BDD and safe to be used. The authors recommend BTX injection to be used as a first-line treatment modality for patients' belly dancer dyskinesia. Even though BTX can be injected directly into abdominal wall, being guided by ultrasound can increases the accuracy of injections site and reduces the risk of complications. ${ }^{13}$ This patient was prescribed with benzodiazepines but she did not use it.

\section{Conclusion}

A pregnant women presented to the clinic and was diagnosed with belly dancer dyskinesia without any underlying pathological findings and it did not occur in the following pregnancy. This is a very rare type of abnormal abdominal movement with unknown etiologic agent, pathogenesis and less known effective treatment. To know the etiologic agent, pathogenesis and effective treatment of similar cases, thorough investigation is important.

\section{Patient Consent}

Written consent has been obtained from the patient after she has seen the detailed description of the case and video to be published. No institutional approval was required for the publishing of this case report.

\section{Acknowledgments}

I want to thank the patient for giving me the consent to publish and Dr Nega Assefa for editing the article.

\section{Funding}

No grant support or funding was given.

\section{Disclosure}

The author has no financial or other disclosures in this study.

\section{References}

1. Criscuolo C, De Leva MF, Sorrentino P, et al. PRICKLE1 progressive myoclonus epilepsy in Southern Italy. Mov Disord. 2010;25 (15):2686-2687. doi:10.1002/mds.23350

2. Aldabbour B, E'leimat I, Alhayek K, Momani A. Recurrent belly dancer's dyskinesia with pregnancy; 2019. Available from: www. e-jmd.org. Accessed December 8, 2021.

3. Meyer JA, Desai KV, Geyer HL. Recurrent belly dancer dyskinesia in pregnancy. Neurology. 2017;88(21):2066. doi:10.1212/WNL.000 0000000003954

4. Iliceto G, Thompson PD, Day BL, Rothwell JC, Lees AJ, Marsden CD. Diaphragmatic flutter, the moving umbilicus syndrome, and "belly dancer's" dyskinesia. Mov Disord. 1990;5(1):15-22. doi:10.1002/mds. 870050105

5. Patil G, Iyer A, Vadgaonkar A, Dalal A, Maydeo A. An unusual cause of abdominal pain. Turk J Gastroenterol. 2020;31(7):547-548. doi:10.5152/tjg.2020.19713 
6. Nigro P, Romoli M, Paolini Paoletti F, Simoni S, Calabresi P, Tambasco N. Levetiracetam-responsive belly dancer's syndrome in a case of progressive supranuclear palsy. Acta Neurol Belg. 2021;121 (3):765-766. doi:10.1007/s13760-020-01343-0

7. Yeh JY, Tu KY, Tseng PT, Lee Y, Lin PY. Acute onset of abdominal muscle dyskinesia ("Belly Dancer Syndrome") from quetiapine exposure: a case report. Clin Neuropharmacol. 2018;41(2):73-74. doi:10.1097/WNF.0000000000000271

8. Roggendorf J, Burghaus L, Liu WC, et al. Belly dancer's syndrome following central pontine and extrapontine myelinolysis. Mov Disord. 2007;22(6):892-894. doi:10.1002/mds.21394

9. Amin OSM, Abdulkarim QH, Shaikhani M. Intermittent bursts of abdominal wall jerky movements: belly dancer's syndrome? $B M J$ Case Rep. 2012;2012:bcr2012007393. doi:10.1136/bcr-2012-007393
10. Inghilleri M, Conte A, Frasca V, Vaudano AE, Meco G. Belly dance syndrome due to spinal myoclonus. Mov Disord. 2006;21 (3):394-396. doi:10.1002/mds.20698

11. Linazasoro G, Van Blercom N, Lasa A, Fernández JM, Aranzábal I. Etiological and therapeutical observations in a case of belly dancer's dyskinesia. Mov Disord. 2005;20(2):251-253. doi:10.1002/ mds. 20312

12. Jabre M, Bejjani BP. Etiological and therapeutical observations in a case of belly dancer's dyskinesia. Mov Disord. 2006;21(9):1536. doi: $10.1002 / \mathrm{mds} .21028$

13. Alshubaili A, Abou-Al-Shaar H, Santhamoorthy P, Attia H, Bohlega S. Ultrasound-guided botulinum toxin A injection in the treatment of belly dancer's dyskinesia. BMC Neurol. 2016;16. doi:10.1186/s12883-016-0746-5

\section{Publish your work in this journal}

The International Medical Case Reports Journal is an international, peer-reviewed open-access journal publishing original case reports from all medical specialties. Previously unpublished medical posters are also accepted relating to any area of clinical or preclinical science. Submissions should not normally exceed 2,000 words or 4 published pages including figures, diagrams and references. The manuscript management system is completely online and includes a very quick and fair peer-review system, which is all easy to use. Visit http://www.dovepress.com/testimonials.php to read real quotes from published authors. 